\title{
Regulation of Enzyme Synthesis in the Arginine Biosynthetic Pathway of Pseudomonas aeruginosa
}

\author{
By R. VOELLMY AND TH. LEISINGER \\ Mikrobiologisches Institut, Eidgenössische Technische Hochschule, \\ ETH-Zentrum, CH-8092 Zürich, Switzerland
}

(Received 11 May 1978; revised 26 June 1978)

In Pseudomonas aeruginosa the synthesis of only two out of eight arginine biosynthetic enzymes tested was regulated. Comparisons were made between the specific activities of these enzymes in bacteria grown on arginine or on its precursor, glutamate. $N^{2}$-Acetylornithine 5-aminotransferase (ACOAT), an enzyme involved in both the biosynthesis and catabolism of arginine, was induced about 14-fold during growth of the organism on arginine as the only carbon and nitrogen source, and the anabolic ornithine carbamoyltransferase (aOTC), a strictly biosynthetic enzyme, was repressed 18-fold. Addition of various carbon sources to the arginine medium led to repression of ACOAT and to derepression of aOTC. Fructose, which supported only slow growth of $P$. aeruginosa, had a weak regulatory effect on the synthesis of the two arginine enzymes while citrate, a good carbon source for this organism, had a strong effect. The repression of ACOAT by citrate was not relieved by adding cyclic AMP to the medium. Under a variety of growth conditions leading to different enzyme activities, a linear relationship between the reciprocal of the specific activity of ACOAT and the specific activity of aOTC was observed. This inverse regulation of the formation of the two enzymes suggested that a single regulatory system governs their synthesis. Such a view was supported by the isolation of citrate-resistant regulatory mutants which constitutively formed ACOAT at the induced level and aOTC at the repressed level.

\section{INTRODUCTION}

Studies on the regulation of amino acid metabolism, conducted with Pseudomonas aeruginosa and $P$. putida, have revealed that these organisms have complex feedback mechanisms regulating the activity of allosteric enzymes in amino acid biosynthetic pathways. Regulation of enzyme synthesis, however, seems to be rare in amino acid biosynthesis whereas it is well documented for several pathways of amino acid catabolism (Clarke \& Ornston, 1975). These generalizations apply to the regulation of arginine metabolism in Pseudomonas (Fig. 1). Inhibition of the first two enzymes of arginine biosynthesis by arginine, by arginine analogues and by several other effectors has been observed with purified enzyme preparations from $P$. aeruginosa (Haas et al., 1972; Leisinger et al., 1974; Haas \& Leisinger, 1975). In the same organism Isaac $\&^{*}$ Holloway (1972) found up to 100 -fold repression of the anabolic ornithine carbamoyltransferase (enzyme 6, Fig. 1) by arginine, whereas the activities of three other arginine biosynthetic enzymes (enzymes 2, 5' and 8, Fig. 1) were not affected by arginine limitation or arginine excess. Voellmy \& Leisinger (1975) reported 15 -fold induction of acetylornithine 5-aminotransferase (enzyme 4, Fig. 1) by arginine. In $P$. putida, $N$-acetylglutamate 5-phosphotransferase and/or $N$-acetylglutamate-5-semialdehyde dehydrogenase (enzymes 2 and 3, Fig. 1) as well as the anabolic ornithine carbamoyltransferase were slightly derepressed during arginine starvation (Condon et al., 1976) and $N^{2}$-acetylornithine 5-aminotransferase was induced by arginine (Chou \& Gunsalus, 


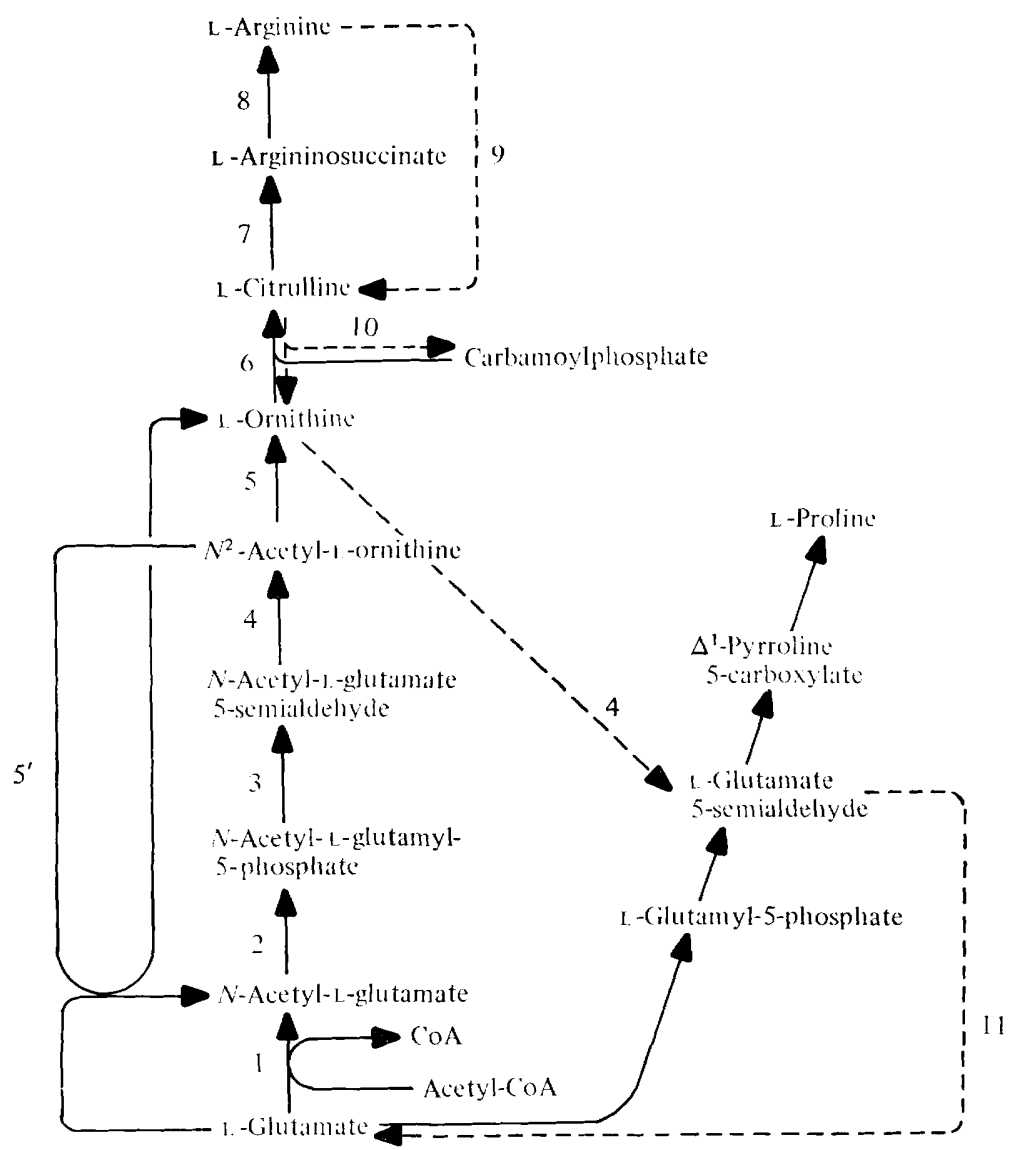

Fig. 1. Arginine metabolism in $P$. aeruginosa. Numbers 1 to 8 designate the arginine biosynthetic enzymes whose trivial and systematic names are listed in Table 1. The dashed line indicates arginine catabolism by the 'deiminase-pathway' involving enzymes: 9, arginine deiminase [L-arginine iminohydrolase; EC 3.5.3.6]; 10, catabolic ornithine carbamoyltransferase [carbamoylphosphate:L-ornithine carbamoyltransferase; EC 2.1.3.3]; 4, [EC 2.6.1.11; see Table 1]; 11, 1-pyrroline dehydrogenase [1-pyrroline-5-carboxylate: $\mathrm{NAD}^{+}$oxidoreductase; EC 1.5.1.12].

1971). In contrast to this limited control of enzyme synthesis in arginine biosynthesis, the enzymes of three different degradative pathways for arginine have all been reported to be inducible by arginine (Clarke \& Ornston, 1975).

In the present study we have confirmed that in $P$. aeruginosa the synthesis of only two out of eight arginine biosynthetic enzymes tested, $N^{2}$-acetylornithine 5-aminotransferase (ACOAT) and the anabolic ornithine carbamoyltransferase (aOTC), is regulated to a marked extent by arginine. The expression of these two enzymes is regulated in a strictly inverse manner by arginine and by different carbon sources, suggesting that a common regulatory system governs their synthesis. Some of the results presented in this paper have been reported in preliminary communications (Voellmy \& Leisinger, 1972, 1976a). 


\section{METHODS}

Bacterial strains. Experiments were done with the wild-type strain Pseudomonas aeruginosa PAo1 (ATCC 15692), the proline-auxotrophic strain PAO951 (pro-137), isolated after mutagenesis of strain PAO1 with 1-methyl-3-nitro-1-nitrosoguanidine, and the citrate-resistant mutant PAO959 (pro-137, CIT-r), which was derived from strain PA0951 by mutagenesis with ethyl methanesulphonate. (The mutation pro-137 causes a defect in the proline-inhibitible ATP:L-glutamate 5-phosphotransferase, the first enzyme of proline biosynthesis; R. V. Krishna, unpublished work.)

Media. Medium P, a nitrogen-free minimal salts medium, was described by Leisinger et al. (1972). Carbon and nitrogen sources were added after sterilization from neutralized stock solutions in various combinations as indicated in Results. Glucose and fructose were used at $28 \mathrm{~mm}, \mathrm{~L}$-amino acids and citrate at $20 \mathrm{~mm}$ and $\left(\mathrm{NH}_{4}\right)_{2} \mathrm{SO}_{4}$ at $15 \mathrm{~mm}$. Solid media were prepared by inclusion of $1.6 \%(\mathrm{w} / \mathrm{v})$ Difco agar.

Growth conditions. Cultures were incubated at $37^{\circ} \mathrm{C}$. Bacteria for enzyme assays were grown in 21 Fernbach flasks containing 11 medium on a rotary shaker $\left(170 \mathrm{rev} . \mathrm{min}^{-1}\right)$ with an eccentricity of $3 \mathrm{~cm}$. They were harvested in the late-exponential phase, washed with saline $[0.85 \%(\mathrm{w} / \mathrm{v}) \mathrm{NaCl}]$ and either used immediately or stored at $-20^{\circ} \mathrm{C}$. Batches of strains PAO951 and PAO959 were routinely tested for the presence of prototrophic revertants by plating samples (approx. $5 \times 10^{9}$ cells) on minimal medium plates. Growth rates were determined with $5 \mathrm{ml}$ cultures in test tubes as described by Leisinger et al. (1974).

Mutant isolation. The isolation of citrate-resistant (CIT-r) mutants was based on the fact that citrate represses ACOAT, which also catalyses the conversion of ornithine to glutamate 5 -semialdehyde (Voellmy \& Leisinger, 1975). Therefore a mutant with an early block in proline synthesis (e.g. PAO951) is unable to derive glutamate 5-semialdehyde from ornithine in the presence of citrate (see Fig. 1) whereas CIT-r derivatives have gained this ability. Spontaneous CIT-r mutants were selected by plating $0.1 \mathrm{ml}$ of a washed suspension $\left(2 \times 10^{8}\right.$ cells $\left.\mathrm{ml}^{-1}\right)$ of strain PA0951 on plates of medium P plus $20 \mathrm{~mm}$-ornithine plus $20 \mathrm{~mm}$-citrate. Alternatively, we treated strain PAO951 with ethyl methanesulphonate as described by Watson \& Holloway (1976) before plating on the selective medium. After incubation for $2 \mathrm{~d}$, large colonies were selected and tested for proline-auxotrophy. Prototrophs (approx. 50\%) were discarded. In the remaining isolates the activity of ACOAT during growth on medium P plus $20 \mathrm{~mm}$-ornithine plus $20 \mathrm{~mm}$-citrate was tested. In about $50 \%$ we detected increased activities of ACOAT. The remaining $50 \%$ showed wild-type ACOAT activities on the ornithine/citrate medium and were discarded.

Preparation of extracts and enzyme assays. The buffers used in the preparation of extracts for the various enzyme assays have been described by Haas et al. (1977). In the experiments reported in this paper we used $0 \cdot 1 \mathrm{M}$-Tris $/ \mathrm{HCl} \mathrm{pH} 7 \cdot 0$ containing $0 \cdot 1 \mathrm{~mm}$-pyridoxal phosphate for the preparation of extracts which were to be assayed for aOTC and ACOAT. Extracts were prepared by suspending wet cells in buffer at $20 \%(\mathrm{w} / \mathrm{v})$ and subjecting them to ultrasonic disruption in an MSE $150 \mathrm{~W}$ ultrasonic disintegrator (eight bursts of $15 \mathrm{~s}$ each). After removal of cell debris by centrifugation at $30000 \mathrm{~g}$ for $20 \mathrm{~min}$, the crude extracts were either dialysed overnight against the extraction buffer or desalted by passage through Sephadex G- 25 .

The methods used for assaying the enzymes of arginine biosynthesis have been listed by Haas et al. (1977). One unit (U) of enzyme is defined as that quantity of enzyme which catalyses the formation of $1 \mu \mathrm{mol}$ of product per $h$ under standard assay conditions. All enzyme activities reported represent the mean from at least two determinations in extracts from independently grown cells. For some experiments an ACOAT assay with a 10-fold increased sensitivity had to be used. It was based on the standard colorimetric assay described by Voellmy \& Leisinger (1975). The incubation mixture (total volume $1.25 \mathrm{ml}$ ) contained: $1.0 \mathrm{ml}$ extract prepared in $0.02 \mathrm{M}$-potassium phosphate buffer $\mathrm{pH} 7.0 ; 130 \mathrm{~mm}$-Tris $/ \mathrm{HCl} \mathrm{pH} 9.0$ at $25^{\circ} \mathrm{C} ; 19 \mathrm{~mm}-N^{2}$-acetylornithine; $19 \mathrm{~mm}$-2-oxoglutarate; $0 \cdot 1 \mathrm{~mm}$-pyridoxal phosphate. The $\mathrm{pH}$ of the reaction mixture containing all components except the enzyme was adjusted to 8.5 at $25^{\circ} \mathrm{C}$. The reaction was started by adding the prewarmed mixture of reagents to the enzyme; after $30 \mathrm{~min}$ incubation at $37^{\circ} \mathrm{C}$ it was stopped by adding

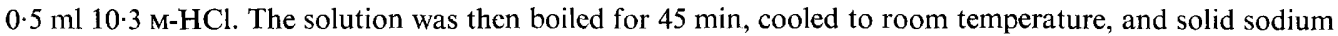
acetate $(1 \mathrm{~g})$ was added to each tube, bringing the total volume of the mixture to $2 \cdot 3 \mathrm{ml}$. Colour was developed by adding $0.2 \mathrm{ml} 33 \mathrm{~mm}-o$-aminobenzaldehyde and $0.35 \mathrm{ml}$ distilled water.

Amidase activity was determined by the transferase assay according to Brammar \& Clarke (1964).

Protein was estimated by the method of Lowry et al. (1951) with bovine serum albumin as a standard. Protein contents of growing bacteria were determined by measuring $A_{546}$ and using an appropriate standard curve.

The sources of the chemicals used were as described by Voellmy \& Leisinger (1975). 
Table 1. Regulation of synthesis of arginine biosynthetic pathway enzymes in $P$. aeruginosa PAOl

Enzyme

\begin{tabular}{|c|c|c|c|}
\hline \multicolumn{2}{|r|}{ Enzyme } & \multicolumn{2}{|c|}{ growth on: } \\
\hline $\begin{array}{l}\text { No. in } \\
\text { Fig. } 1\end{array}$ & Trivial and systematic name & $\begin{array}{l}\text { Medium P+ } \\
\text { glutamate* }\end{array}$ & $\begin{array}{l}\text { Medium } \mathbf{P}+ \\
\text { arginine* }\end{array}$ \\
\hline 1 & $\begin{array}{l}N \text {-Acetylglutamate synthase [acetyl-CoA: } \\
\text { L-glutamate } N \text {-acetyltransferase; EC } 2.3 .1 .1]\end{array}$ & $0.28 \dagger$ & $0 \cdot 28 \uparrow$ \\
\hline ? & $\begin{array}{l}N \text {-Acetylglutamate 5-phosphotransferase } \\
\text { [ATP: } N \text {-acetyl-L-glutamate } 5 \text {-phosphotransferase; } \\
\text { EC } 2.7 .2 .8 \text { ] }\end{array}$ & $1.92 \ddagger$ & $1.86 \%$ \\
\hline 3 & $\begin{array}{l}N \text {-Acetylglutamate-5-semialdehyde dehydrogenase } \\
\text { [ } N \text {-acetyl-L-glutamate-5-semialdehyde:NADP }{ }^{+} \\
\text {oxidoreductase (phosphorylating); EC } 1.2 .1 .38]\end{array}$ & $1 \cdot 40$ & $1 \cdot 15$ \\
\hline 4 & $\begin{array}{l}N^{2} \text {-Acetylornithine 5-aminotransferase (ACOAT) } \\
{\left[N^{2} \text {-acetyl-L-ornithine: } 2 \text {-oxoglutarate }\right.} \\
\text { aminotransferase; EC } 2.6 .1 .11 \text { ) }\end{array}$ & $4 \cdot 8$ & 73.00 \\
\hline 5 & $\begin{array}{l}\text { Acetylornithinase }\left[N^{2} \text {-acetyl-L-ornithine }\right. \\
\text { amidohydrolase; EC 3.5.1.16] }\end{array}$ & $0 \cdot 58$ & $0 \cdot 53$ \\
\hline $5^{\prime}$ & $\begin{array}{l}\text { Ornithine acetyltransferase }\left[N^{2} \text {-acetyl-L- }\right. \\
\text { ornithine:L-glutamate } N \text {-acetyltransferase; } \\
\text { EC 2.3.1.35] }\end{array}$ & $0 \cdot 44$ & 0.48 \\
\hline 6 & $\begin{array}{l}\text { Anabolic ornithine carbamoyltransferase (aOTC) } \\
\text { [carbamoylphosphate: L-ornithine } \\
\text { carbamoyltransferase; EC } 2.1 .3 .3 \text { ] }\end{array}$ & $4 \cdot 79$ & $0 \cdot 27$ \\
\hline 7 & $\begin{array}{l}\text { Argininosuccinate synthetase [L-citrulline: } \\
\text { L-aspartate ligase (AMP-forming); EC 6.3.4.5] }\end{array}$ & ND & ND \\
\hline 8 & $\begin{array}{l}\text { Argininosuccinase [L-argininosuccinate } \\
\text { arginine-lyase; EC } 4.3 .2 .1 \text { ] }\end{array}$ & 0.22 & $0 \cdot 15$ \\
\hline
\end{tabular}

Specific enzyme activity

[U (mg protein) ${ }^{-1}$ ] after growth on:

\section{RESULTS}

The synthesis of two arginine biosynthetic enzymes is regulated by arginine

When the wild-type strain PAOl was grown on medium $P$ with either arginine or glutamate as the only carbon and nitrogen source, we observed about 14-fold induction of ACOAT and about 18-fold repression of aOTC by arginine (Table 1). The activities of enzymes 3 and 8 (see Fig. 1) were repressed very slightly, but reproducibly, by arginine whereas the activities of enzymes 1, 2, 5 and $5^{\prime}$ were not altered (Table 1). As a control, a double auxotrophic strain with a requirement for leucine and lesions in enzymes 2 and 7 of the arginine pathway was grown in a chemostat (growth rate $0.2 \mathrm{~h}^{-1}$ ) on minimal medium $\mathrm{E}$ (Vogel \& Bonner, 1956) either under leucine limitation with an excess of arginine (repressive conditions) or under arginine limitation with an excess of leucine (derepressive conditions). Among seven arginine enzymes tested, the activities of only ACOAT and aOTC were altered by a factor of greater than two in response to changes in the arginine supply. It thus seemed unlikely that the lack of derepression of most of the arginine biosynthetic enzymes on medium $P$ with glutamate was due to a high concentration of endogenously formed arginine.

The induction of ACOAT by arginine has been explained earlier by the dual role of this enzyme in arginine biosynthesis and in the catabolism of arginine via ornithine and glutamate 5-semialdehyde (Voellmy \& Leisinger, 1975, 1976 b). In order to exclude the possibility that there are two ACOATs, one biosynthetic and one degradative, we compared the chromatographic properties, the sedimentation behaviour in sucrose gradients, the electrophoretic mobility, the substrate specificity, and the inhibition pattern by substrate analogues 


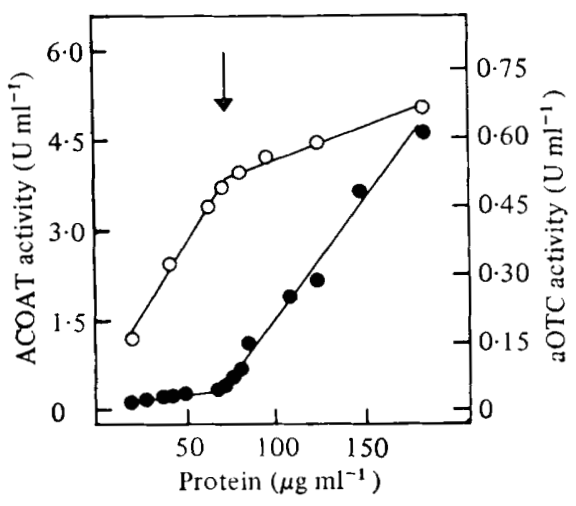

Fig. 2. Induction of ACOAT and repression of aOTC by arginine. Strain PAO1 was grown in a Fernbach flask on medium $P$ with $20 \mathrm{~mm}$-glutamate. At intervals, $5 \mathrm{ml}$ samples were removed for the determination of ACOAT, aOTC and protein. ACOAT was determined by the assay procedure with a 10-fold increased sensitivity (see Methods). The arrow indicates the addition of arginine to the growing culture up to a final concentration of $20 \mathrm{~mm} . \bullet, A C O A T$; $\bigcirc$, aOTC.

of ACOAT from arginine-grown cells with ACOAT from glutamate-grown cells. There were no detectable differences between the enzymes from the two sources (R. Voellmy and Th. Leisinger, unpublished work). We thus conclude that $P$. aeruginosa, in contrast to Klebsiella aerogenes (Friedrich et al., 1978), forms only one ACOAT. Induction of ACOAT by arginine was not restricted to $P$. aeruginosa PAO; we have also observed a 15 -fold induction of the enzyme by arginine in $P$. aeruginosa strains PAC and PAT.

The differential rates of synthesis of both ACOAT and aOTC changed immediately on addition of arginine to a culture growing on medium $P$ with 20 mm-glutamate (Fig. 2). When chloramphenicol at $500 \mu \mathrm{g} \mathrm{ml}^{-1}$ was added $1 \mathrm{~min}$ prior to arginine, the activities of ACOAT and aOTC remained unchanged for at least $3 \mathrm{~h}$. These observations indicated that the two enzymes were metabolically stable and that the changes observed in their activities on addition of arginine were indeed due to induction and repression respectively.

\section{Regulation of enzyme synthesis by carbon sources is superimposed on arginine-specific regulation}

The specific activity of ACOAT was lower in cultures grown in a medium containing arginine plus an additional carbon source than in a culture grown on arginine alone (Table 2). $\left(\mathrm{NH}_{4}\right)_{2} \mathrm{SO}_{4}$ was included in all media in this series of experiments to eliminate possible regulatory effects caused by the availability of nitrogen. The severity of the repression of ACOAT by carbon compounds was not strictly correlated with the specific growth rate supported by these compounds when present as the only sources of carbon and energy. Fructose supported only slow growth of $P$. aeruginosa and caused weak repression of ACOAT. Citrate, glutamate and glucose were all good carbon sources, allowing growth rates of approximately $0.8 \mathrm{~h}^{-1}$, but repressed to different extents. Proline was an intermediate carbon source but repressed the synthesis of ACOAT very effectively. The variety of compounds exerting repression of ACOAT (Table 2) suggested that they did not interfere specifically with ACOAT synthesis but were metabolized and thereby led to catabolite repression.

An analysis of the kinetics of citrate-repression of ACOAT revealed some analogies to the phenomenon of catabolite repression as described in the $\beta$-galactosidase system of Escherichia coli (Magasanik, 1970) and in the amidase system of $P$. aeruginosa (Smyth $\&$ Clarke, 1975). Immediately after addition of citrate to a culture growing on arginine plus $\left(\mathrm{NH}_{4}\right)_{2} \mathrm{SO}_{4}$, the differential rate of ACOAT synthesis went through a phase of severe transient repression which!lasted for about two doublings (Fig. 3a). After this period, enzyme synthesis 


\section{Table 2. Repression of ACOAT by carbon sources}

Strain PAO1 was grown in medium $\mathrm{P}$ with $15 \mathrm{~mm}-\left(\mathrm{NH}_{4}\right)_{2} \mathrm{SO}_{4}$ and the various additions indicated. ACOAT activities and growth rates were determined as described in Methods.

Addition(s)*

Arginine

Arginine + Fructose

Arginine + Glutamate

Arginine + Glucose

Arginine + Proline

Arginine + Citrate

Fructose

Glutamate

Glucose

Proline

Citrate

$$
\begin{gathered}
\text { Specific activity } \\
\text { of ACOAT }
\end{gathered}
$$

[U (mg protein $)^{-1}$ ]

$72 \cdot 0$
$51 \cdot 6$
$43 \cdot 8$
$34 \cdot 8$
$24 \cdot 6$
$14 \cdot 4$
$4 \cdot 0$
$4 \cdot 0$
$4 \cdot 3$
$3 \cdot 4$
$3 \cdot 2$

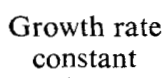

Growth rate constant

$\left(\mathrm{h}^{-1}\right)$

\subsection{8}

0.55

0.80

0.80

0.65

0.80

$0 \cdot 25$

0.80

0.80

0.55

0.80

* All $20 \mathrm{~mm}$, except glucose and fructose which were added at $28 \mathrm{~mm}$.
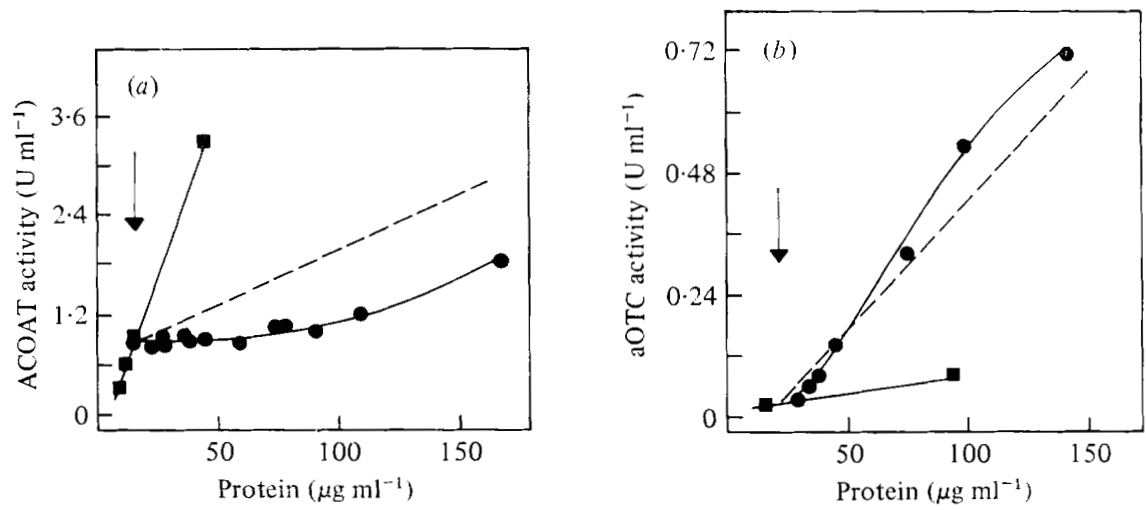

Fig. 3. Regulation of the synthesis of ACOAT and aOTC by citrate. Two cultures of strain PAO1 were grown in Fernbach flasks on medium $\mathrm{P}$ with $15 \mathrm{~mm}-\left(\mathrm{NH}_{4}\right)_{2} \mathrm{SO}_{4}$ and $20 \mathrm{~mm}$-arginine. For other details, see legend to Fig. 2. The arrow indicates the addition of citrate up to a final concentration of $20 \mathrm{~mm}$ to one of the cultures (O); the second culture ( $\boldsymbol{\square})$ served as a control. The dashed line represents the steady state rate of enzyme formation on medium $\mathrm{P}$ with $15 \mathrm{~mm}-\left(\mathrm{NH}_{4}\right)_{2} \mathrm{SO}_{4}, 20 \mathrm{~mm}-$ arginine and $20 \mathrm{~mm}$-citrate. Differential rates of formation of $\operatorname{ACOAT}(a)$ and aOTC $(b)$ were measured in separate experiments.

proceeded at the rate characteristic of the new composition of the growth medium. In contrast to the observations in the amidase system of $P$. aeruginosa, addition of cyclic AMP up to $40 \mathrm{~mm}$ relieved neither the transient nor the permanent repression of ACOAT by citrate. The weak repression mediated by $28 \mathrm{~mm}$-glucose or by $20 \mathrm{~mm}$-glutamate was also insensitive to cyclic AMP. Substitution of dibutyryl-cyclic AMP was without effect.

In similar experiments we observed derepression of aOTC by citrate (Fig. $3 b$ ).

\section{$A C O A T$ and aOTC are inversely regulated under various growth conditions}

There was a linear relationship between the reciprocal of the specific activity of ACOAT and the specific activity of aOTC (Fig. 4) in cells that had been grown in different media. However, in the experiments described so far, the growth rate and thereby the metabolic state of the cells changed from one set of growth conditions to another. It was therefore not clear whether the inverse regulation of ACOAT and aOTC was brought about by a common arginine-specific regulatory system governing the expression of both enzymes and/or by the 


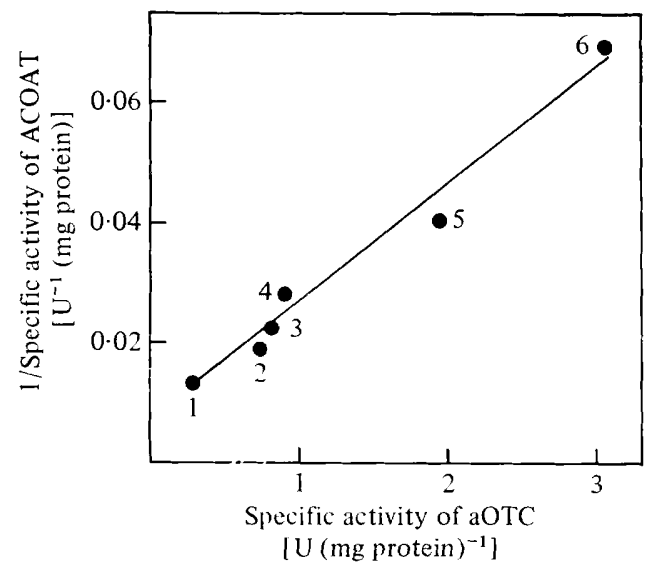

Fig. 4. Inverse regulation of the synthesis of ACOAT and aOTC by different carbon sources. Strain PAO1 was grown on medium $\mathrm{P}$ with $15 \mathrm{~mm}-\left(\mathrm{NH}_{4}\right)_{2} \mathrm{SO}_{4}$ and $20 \mathrm{~mm}$-arginine. The effect of various additions to this medium on the specific activities of ACOAT and aOTC was determined: 1 , no addition; 2, 28 mm-fructose; 3, 20 mm-glutamate; 4, 28 mm-glucose; 5, 20 mM-proline; 6, $20 \mathrm{mm-}$ citrate.

catabolite repression system acting in an opposite manner on the synthesis of the two proteins. We therefore developed a procedure by which the uptake of arginine was restricted without changing the growth rate.

Lysine is a poor growth substrate for P. aeruginosa wild type (Fothergill \& Guest, 1977). In strain PAO1 it supports a mean generation time of about $11 \mathrm{~h}$ when present as the only carbon and nitrogen source in medium P. Lysine also competes with arginine for one of the two transport systems for arginine in P. putida (Fan et al., 1972). We could therefore restrict uptake of arginine by adding lysine to the growth medium. The growth rate of strain PAOl on medium $\mathrm{P}$ with arginine and $\left(\mathrm{NH}_{4}\right)_{2} \mathrm{SO}_{4}$ was progressively inhibited as the concentration of lysine in the medium was increased (Fig. 5). The growth rate of cultures on $20 \mathrm{~mm}-$ glutamate, $10 \mathrm{~mm}$-arginine and $15 \mathrm{~mm}-\left(\mathrm{NH}_{4}\right)_{2} \mathrm{SO}_{4}$, however, was not affected by the addition of lysine up to $120 \mathrm{~mm}$. The activities of ACOAT and aOTC determined in cells which had been grown on the latter medium at the same rate but with different lysine concentrations showed inverse regulation of the two enzymes. The enzyme activities depended on the degree of arginine restriction caused by different lysine concentrations during growth (Fig. 6). This experiment suggests that the inverse regulation of ACOAT and aOTC depends on the intracellular concentration of arginine, which might act as a corepressor in a regulatory system controlling the synthesis of the two enzymes. It is not clear at this stage whether carbon sources regulate the expression of the two arginine enzymes indirectly by decreasing the intracellular concentration of arginine or directly by triggering changes in the intracellular concentration of an effector which forms part of a catabolite repression system.

\section{Citrate-resistant mutants}

Ten CIT-r derivatives of strain PA0951 were isolated. When they were grown in medium $\mathbf{P}$ with ornithine and citrate, their ACOAT activities were 18- to 60-fold higher than the ACOAT activities of the wild type grown under the same conditions. All CIT-r mutants grew extremely slowly on medium P with ornithine and, like strain PA0951, failed to grow on medium $\mathbf{P}$ with arginine. Ornithine thus satisfies the proline requirement of strain PAO951 while arginine, although being catabolized via ornithine, is unable to do so. At present we can explain neither the inability of CIT-r mutants to grow on ornithine nor the lack of growth of strain PA0951 on arginine.

One CIT-r mutant, strain PAO959, was compared with the wild type and with its parent, 


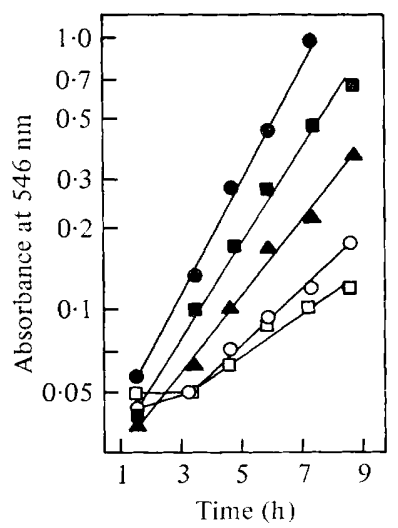

Fig. 5

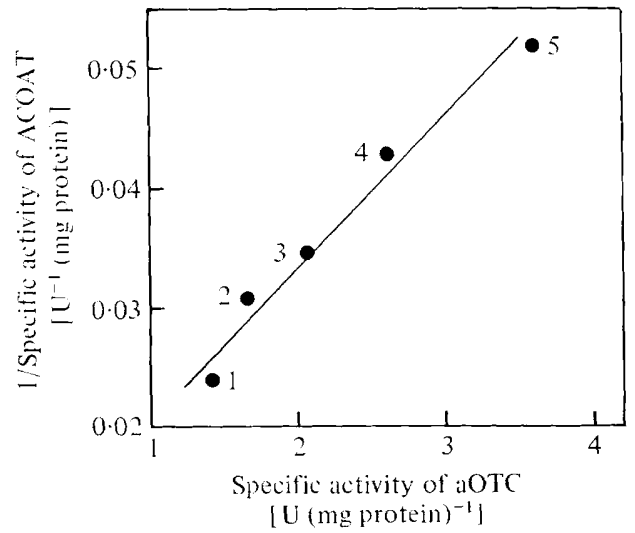

Fig. 6

Fig. 5. Inhibition by lysine of growth on arginine. Strain PAO1 was grown in Fernbach flasks on medium $\mathrm{P}$ with $15 \mathrm{~mm}-\left(\mathrm{NH}_{4}\right)_{2} \mathrm{SO}_{4}$ and $10 \mathrm{~mm}$-arginine. The effect of increasing concentrations of lysine on the growth rate was determined: $\boldsymbol{\bullet}$, no addition; $\boldsymbol{\Delta}, 40 \mathrm{mM}-\mathrm{L}-\mathrm{lysine} ; \boldsymbol{\Delta}, 80 \mathrm{~mm}$-L-lysine; $\bigcirc, 120 \mathrm{~mm}$-L-lysine; $\square, 200 \mathrm{~mm}$-L-lysine.

Fig. 6. Inverse regulation of the synthesis of ACOAT and aOTC by arginine. Strain PAol was grown in medium $\mathrm{P}$ with $15 \mathrm{~mm}-\left(\mathrm{NH}_{4}\right)_{2} \mathrm{SO}_{4}, 20 \mathrm{~mm}$-glutamate and $10 \mathrm{~mm}$-arginine. The effect of different degrees of arginine restriction by lysine on the specific activities of ACOAT and aOTC was determined: 1, no addition; 2, $10 \mathrm{~mm}$-L-lysine; 3, $20 \mathrm{mM}$-L-lysine; 4, $40 \mathrm{~mm}$-L-lysine ; 5, $80 \mathrm{~mm}$-Llysine.

Table 3. Specific activity of ACOAT and aOTC in the wild type (PAO1), the citrate-resistant mutant (PA0959) and its parent (PA0951) under different conditions of growth

Growth, preparation of extracts and determination of enzyme activities are described in Methods. The extremely slow growth of strain PAO959 in medium P with ornithine led to the selection of citrate-sensitive revertants, so it was not possible to obtain ornithine-grown cells for enzyme assays. Each value represents the arithmetic mean \pm standard deviation of three independent determinations.

$\quad$ Addition(s)* to
$\quad$ medium $\mathbf{P}$
Arginine
Glutamate
Proline
Proline + Citrate
Ornithine
Ornithine + Citrate

ACOAT activity [U (mg protein $\left.)^{-1}\right]$

$\begin{array}{rccccc}\text { PAO1 } & \text { PAO951 } & \text { PAO959 } & \text { PAO1 } & \text { PAO951 } & \text { PAO959 } \\ 73 \cdot 0 \pm 11 \cdot 0 & \text { NG } & \text { NG } & 0 \cdot 27 \pm 0 \cdot 10 & \text { NG } & \text { NG } \\ 6 \cdot 2 \pm 1 \cdot 1 & \text { NG } & \text { NG } & 4 \cdot 78 \pm 1 \cdot 41 & \text { NG } & \text { NG } \\ 5 \cdot 7 \pm 1 \cdot 8 & 5 \cdot 8 \pm 2 \cdot 0 & 66 \cdot 6 \pm 8 \cdot 7 & 2 \cdot 74 \pm 0 \cdot 48 & 5 \cdot 04 \pm 0.99 & 0 \cdot 60 \pm 0 \cdot 1 \\ 5 \cdot 5 \pm 0 \cdot 4 & 4 \cdot 6 \pm 0 \cdot 1 & 83 \cdot 6 \pm 7 \cdot 4 & 3 \cdot 45 \pm 0 \cdot 10 & 5 \cdot 67 \pm 0.44 & 0 \cdot 74 \pm 0 \cdot 2 \\ 34 \cdot 0 \pm 4 \cdot 6 & 13 \cdot 3 \pm 0 \cdot 7 & - & 0 \cdot 64 \pm 0 \cdot 24 & 1 \cdot 04 \pm 0 \cdot 17 & - \\ 11 \cdot 2 \pm 1 \cdot 1 & \text { NG } & 120 \cdot 8 \pm 13 \cdot 3 & 2 \cdot 05 \pm 0 \cdot 16 & \text { NG } & 2 \cdot 35 \pm 0.5\end{array}$

NG, No growth. * All $20 \mathrm{~mm}$.

strain PA0951 (Table 3). During growth on medium $\mathbf{P}$ with proline, the mutant had an induced level of ACOAT activity and a partially repressed level of aOTC activity whereas strains PAOl and PAO951 showed non-induced levels of ACOAT and derepressed levels of aOTC. In the CIT-r mutant ACOAT synthesis had become insensitive to repression by citrate while aOTC was still derepressed by the addition of citrate to a medium containing ornithine as the only carbon and nitrogen source but was insensitive to citrate-derepression in the proline medium. Addition of $\left(\mathrm{NH}_{4}\right)_{2} \mathrm{SO}_{4}$ to the ornithine/citrate medium had no effect on the activity of aOTC in strain PAO959. Thus the mutation to citrate resistance affected the arginine-specific control of ACOAT and aOTC synthesis and at the same time abolished the regulation of the synthesis of ACOAT by citrate. In the absence of a genetic analysis of strain PAO959 it cannot be excluded that the phenotypic properties of the strain are due to a double mutation. However, the high rate at which CIT-r mutants arise and the fact that the ACOAT 
and aOTC activities in other CIT-r mutants were similar to the activities in strain PAO959 do not support this possibility. The rate of synthesis of acetamide amidase, another inducible enzyme regulated by catabolite repression (Smyth \& Clarke, 1975), was still sensitive to repression by citrate in strain PAO959. As in the wild type the induced rate of amidase synthesis was repressed by approximately $40 \%$ on addition of $10 \mathrm{~mm}$-citrate. It is therefore unlikely that the resistance of ACOAT to citrate repression was due to a change in citrate metabolism leading to a generalized resistance to catabolite repression.

\section{DISCUSSION}

Pseudomonas aeruginosa synthesizes arginine from glutamic acid via ornithine and citrulline in eight enzymic steps. The transacetylase modification of the arginine pathway (Fig. 1) provides two enzymes for the formation of ornithine from $N^{2}$-acetylornithine: ornithine acetyltransferase and acetylornithinase. Six of the nine loci coding for the arginine biosynthetic enzymes have been mapped and are scattered in the 18 to $55 \mathrm{~min}$ region of the chromosome of strain PAO (Haas et al., 1977). Arginine is also efficiently degraded by $P$. aeruginosa. In contrast to the situation in the biosynthetic pathway, the genetics and the regulation of arginine catabolism are largely unknown. The enzymes present in strain PAO suggest at least two different pathways for the catabolism of arginine: the 'deiminasepathway' via citrulline, ornithine and glutamate 5-semialdehyde (Fig. 1) and the "decarboxylase-pathway' leading to agmatine, putrescine and 4-aminobutyrate. In an earlier communication we reported that a mutant lacking ACOAT grows very slowly on arginine and not at all on ornithine (Voellmy \& Leisinger, 1976 b) and therefore concluded that the 'deiminase-pathway' was the preferred route of arginine degradation under the growth conditions we used.

If this is the case, $P$. aeruginosa is faced with the problem of regulating the flow of metabolites participating in opposing pathways. As ornithine and citrulline are intermediates both of arginine biosynthesis and of arginine catabolism, an energy-wasting cycle of arginine breakdown and resynthesis must be avoided during growth on arginine. In Saccharomyces cerevisiae, which has a urea cycle, the control of opposing pathways of arginine metabolism has been studied in great detail by Wiame and collaborators (Wiame \& Dubois, 1976). They have detected an arginine-specific regulatory system controlling the synthesis of the anabolic and the catabolic urea cycle enzymes in an inverse manner and in addition an inactivation of aOTC by formation of a complex with arginase in the presence of arginine.

The present survey of the regulation of synthesis of eight arginine biosynthetic enzymes in $P$. aeruginosa has confirmed that the synthesis of only two enzymes is effectively regulated by arginine: aOTC, the enzyme processing ornithine in the biosynthetic direction, is repressed about 18 -fold by arginine and 7 -fold by ornithine, whereas ACOAT, an enzyme which, in addition to its biosynthetic function, catabolizes ornithine to glutamate 5-semialdehyde, is induced 14-fold by arginine and 6-fold by ornithine. Ornithine thus occupies a key position in arginine metabolism and is channelled into either the catabolic or the biosynthetic pathway according to the arginine status of the cell. As arginine and ornithine are interconvertible, we do not know whether both compounds, only one of them, or a metabolite derived from them is the true effector in the regulatory system. One would expect a similar mechanism preventing a futile cycle between citrulline and arginine. Whereas the catabolic ornithine carbamoyltransferase is induced by arginine (Stalon et al., 1972), we lack information on the regulation of argininosuccinate synthetase by arginine. The aOTC of $P$. fluorescens has been shown to form a dead-end complex with citrulline (Stalon et al., 1977). High concentrations of citrulline formed from arginine might thus inhibit aOTC thereby leaving ornithine exclusively accessible to the catabolic reaction under conditions of arginine excess. It is possible that this mechanism and other, as yet unknown, mechanisms acting at the level of enzyme activity also exist in $P$. aeruginosa and contribute to the maintenance of a 
balance between arginine synthesis and arginine breakdown under various conditions of growth.

The regulation by carbon sources of the two enzymes whose synthesis is controlled by arginine and ornithine seems physiologically important in the case of ACOAT but is not readily understandable for aOTC. There is no obvious metabolic need to increase the rate of synthesis of an arginine biosynthetic enzyme in the presence of arginine and citrate over the rate observed on arginine alone. The inverse regulation of ACOAT and aOTC by carbon sources could be due to inhibition of arginine uptake by citrate and other carbon sources. This explanation was offered by Isaac \& Holloway (1972) who observed that arginine repressed the aOTC of $P$. aeruginosa PAOl most efficiently when present as the sole carbon source. Alternatively the regulation of the two ornithine-utilizing enzymes by carbon sources might be due to a catabolite repression system which is independent of the arginine regulatory mechanism. In $P$. aeruginosa this type of situation has been observed for two histidineutilizing enzymes (Potts \& Clarke, 1976) and for the aliphatic amidase (Smyth \& Clarke, 1975).

Since our physiological experiments did not allow a conclusion as to which one of the two hypotheses was more likely to explain the regulatory phenomena in the arginine system, we isolated mutants (CIT-r) with defective regulation. The properties of one of these, strain PAO959, suggest that one mutational event has completely eliminated the arginine-specific regulation and at the same time has severely altered citrate-regulation of both enzymes. We therefore favour the idea that the inverse regulation of ACOAT and aOTC is due to a specific regulatory system common to both enzymes and that the inducer/corepressor concentration in this system is modulated by citrate and other carbon sources via an entry exclusion mechanism.

The synthesis of several amino acid-catabolizing enzymes has been reported to be governed not only by induction and catabolite repression but also by nitrogen repression. This threefold control of enzyme formation has recently been demonstrated for ACOAT of Klebsiella aerogenes (Friedrich et al., 1978) and for histidase and urocanase of $P$. aeruginosa (Potts \& Clarke, 1976). In the present study we observed no effect of $\left(\mathrm{NH}_{4}\right)_{2} \mathrm{SO}_{4}$ on the repression of ACOAT by citrate. Citrate and other carbon sources repressed the induced synthesis of ACOAT whether $\left(\mathrm{NH}_{4}\right)_{2} \mathrm{SO}_{4}$ was present in the medium or not. This observation may either reflect the absence of nitrogen regulation in the control of ACOAT synthesis or it may be due to the generation from the inducer arginine of non-limiting concentrations of ammonium by arginine deiminase and catabolic ornithine carbamoyltransferase. The latter possibility seems likely as the addition of $\left(\mathrm{NH}_{4}\right)_{2} \mathrm{SO}_{4}$ had no effect on the growth rate of strain PAOl on a medium with arginine as the only carbon and nitrogen source. In order to test the involvement of nitrogen repression in the ACOAT/aOTC system it will therefore be necessary to study the regulation of these enzymes under nitrogen limitation in mutants with a defective arginine transport system or defective arginine catabolism.

We wish to thank Ursula Bodmer for technical assistance. This study was supported by the Swiss National Foundation for Scientific Research (project no. 3.5090.75).

\section{REFERENCES}

Brammar, W. J. \& Clarke, P. H. (1964). Induction and repression of Pseudomonas aeruginosa amidase. Journal of General Microbiology 37, 307-319.

Chou, I. N. \& Gunsalus, I. C. (1971). Arginine biosynthesis in Pseudomonas putida. Bacteriological Proceedings, 162.

Clarke, P. H. \& Ornston, L. N. (1975). In Genetics and Biochemistry of Pseudomonas, pp. 191-340.
Edited by P.H. Clarke \& M. H. Richmond. London: Wiley.

Condon, S., Collins, J. K. \& O'Donovan, G. A. (1976). Regulation of arginine and pyrimidine biosynthesis in Pseudomonas putida. Journal of General Microbiology 92, 375-383.

Fan, C. L., Miller, C. L. \& Rodwell, V.W. (1972). Metabolism of basic amino acids in Pseudomonas putida. Transport of lysine, ornithine, 
and arginine. Journal of Biological Chemistry 247, 2283-2288.

Fothergill, J. C. \& Gúnst, J. R. (1977). Catabolism of L-lysine by Pseudomonas aeruginosa. Journal of General Microbiology 99, 139-155.

Friedrich, B., Friedrich, C. G. \& Magasanik, B. (1978). Catabolic $N^{2}$-acetylornithine 5-aminotransferase of Klebsiella aerogenes: control of synthesis by induction, catabolite repression, and activation by glutamine synthetase. Journal of Bacteriology 133, 686-691.

HAAS, D. \& LeISINGER, T. (1975). $N$-Acetylglutamate 5-phosphotransferase of Pseudomonas aeruginosa. Catalytic and regulatory properties. European Journal of Biochemistry 52, 377-383.

HaAs, D., Kurer, V. \& Leisinger, T. (1972). $N$-Acetylglutamate synthetase of Pseudomonas aeruginosa. An assay in vitro and feedback inhibition by arginine. European Journal of Biochemistry 31, 290-295.

Haas, D., Holloway, B. W., Schamboeck, A. \& LEISINGER, Th. (1977). The genetic organization of arginine biosynthesis in Pseudomonas aeruginosa. Molecular and General Genetics 154, 7-22.

IsAaC, J. H. \& Holloway, B. W. (1972). Control of arginine biosynthesis in Pseudomonas aeruginosa. Journal of General Microbiology 73, 427-438.

Leisinger, Th., HaAs, D. \& Hegarty, M. P. (1972). Indospicine as an arginine antagonist in Escherichia coli and Pseudomonas aeruginosa. Biochimica et biophysica acta 262, 214-219.

Leisinger, Th., O'Sullivan, CH. \& HaAs, D. (1974). Arginine analogues: effect on growth and on the first two enzymes of the arginine pathway in Pseudomonas aeruginosa. Journal of General Microbiology 84, 253-260.

Lowry, O. H., Rosebrough, N. J., Farr, A. L. \& RANDALL, R. J. (1951). Protein measurement with the Folin phenol reagent. Journal of Biological Chemistry 193, 265-275.

MagasaniK, B. (1970). In The Lactose Operon, 1st edn, pp. 189-219. Edited by J. R. Beckwith \& D. Zipser. Cold Spring Harbor: Cold Spring Harbor Laboratory.

Potts, J. R. \& Clarke, P. H. (1976). The effect of nitrogen limitation on catabolite repression of amidase, histidase and urocanase in Pseudomonas aeruginosa. Journal of General Microbiology 93, 377-387.

Smyth, P. F. \& Clarke, P. H. (1975). Catabolite repression of Pseudomonas aeruginosa amidase: the effect of carbon source on amidase synthesis. Journal of General Microbiology 90, 81-90.

Stalon, V., Ramos, F., Piérard, A. \& Wiame, J. M. (1972). Regulation of the catabolic ornithine carbamoyltransferase of Pseudomonas fluorescens. European Journal of Biochemistry 29, 25-35.

Stalon, V., Legrain, Ch. \& Wiame, J. M. (1977). Anabolic ornithine carbamoyltransferase of Pseudomonas. The bases of its functional specialization. European Journal of Biochemistry 74, 319-327.

Voellmy, R. \& Leisinger, Th. (1972). Regulation of enzyme synthesis in the arginine system of Pseudomonas aeruginosa. Journal of General Microbiology 73, xiii.

Voellmy, R. \& Leisinger, Th. (1975). Dual role for $\mathrm{N}^{2}$-acetylornithine 5-aminotransferase from Pseudomonas aeruginosa in arginine biosynthesis and arginine catabolism. Journal of Bacteriology 122, 799-809.

Voellmy, R. \& Leisinger, Th. (1976a). Common control of a biosynthetic and a degradative enzyme of arginine in Pseudomonas aeruginosa. Bacteriological Proceedings, 156.

Voellmy, R. \& Leisinger, Th. (1976 b). Role of 4-aminobutyrate aminotransferase in the arginine metabolism of Pseudomonas aeruginosa. Journal of Bacteriology 128, 722-729.

Vogel, H. J. \& BonNer, D. M. (1956). Acetylornithinase of Escherichia coli: partial purification and some properties. Journal of Biological Chemistry 218, 97-106.

Watson, J. M. \& Holloway, B. W. (1976). Suppressor mutations in Pseudomonas aeruginosa. Journal of Bacteriology 125, 780-786.

Wiame, J. M. \& Dưois, E. L. (1976). In Second International Symposium on the Genetics of Industrial Microorganisms, 1st edn, pp. 391-406. Edited by K. D. Macdonald. London: Academic Press. 